\title{
Endoscopic surveillance of head and neck cancer in patients with esophageal squamous cell carcinoma
}

Authors

Institution
Minoru Kato, Ryu Ishihara, Kenta Hamada, Yusuke Tonai, Yasushi Yamasaki, Noriko Matsuura, Takashi Kanesaka, Sachiko Yamamoto, Tomofumi Akasaka, Noboru Hanaoka, Yoji Takeuchi, Koji Higashino, Noriya Uedo, Hiroyasu lishi

Department of Gastrointestinal Oncology, Osaka Medical Center for Cancer and Cardiovascular Diseases, Osaka, Japan submitted 21. January 2016 accepted after revision 11. April 2016

\section{Bibliography}

DOI http://dx.doi.org/

10.1055/s-0042-106720

Published online: 21.6.2016

Endoscopy International Open 2016; 04: E752-E755

(c) Georg Thieme Verlag KG Stuttgart · New York

E-ISSN 2196-9736

\section{Corresponding author}

\section{Ryu Ishihara, MD}

Department of Gastrointestinal Oncology

Osaka Medical Center for

Cancer and Cardiovascular Diseases

1-3-3 Nakamichi Higashinari-ku, Osaka 537-8511

Japan

Phone: +81-6-6972-1181

Fax: +81-6-6981-4067

isihara-ry@mc.pref.osaka.jp
Background and study aims: Multiple squamous cell carcinomas (SCCs) frequently arise in the upper aerodigestive tract, referred to as the field cancerization phenomenon. The aim of this study was to elucidate the detailed clinical features of second primary head and neck (H\&N) SCCs arising in patients with esophageal SCC.

Patients and methods: A total of 818 patients underwent endoscopic resection for superficial esophageal cancer between January 2006 and December 2013. Of these, 439 patients met our inclusion criteria, and we retrospectively investigated the incidence, primary sites, and stages of second primary H\&N SCCs in these patients.

Results: A total of 53 metachronous H\&N SCCs developed in 40 patients after a median follow-up period of 46 months (range 9-109). The cumula-

\section{Introduction}

\section{$\nabla$}

Multiple squamous cell carcinomas (SCCs) frequently arise in the upper aerodigestive tract [1, 2]. This phenomenon can be explained by the field cancerization theory, which suggests that the entire epithelial surface of the upper aerodigestive tract is at increased risk of developing cancers because of its repeated exposure to carcinogens such as alcohol and through smoking $[3,4]$. Surveillance of the aerodigestive tract in patients with head and neck (H\&N) and esophageal SCCs is therefore recommended based on this theory, and periodic endoscopic examinations in these patients may facilitate the early detection of SCCs in this area [5]. Early detection of cancers may be associated with a better prognosis and increased possibility of minimally invasive treatment, including endoscopic resection [6-8].

Although previous studies have reported on the use of surveillance endoscopic examinations in patients with esophageal cancer [9-11], they have only included small numbers of patients, and no detailed information on the second pri- tive incidence rates of metachronous H\&N SCCs at 3,5 , and 7 years were $5.3 \%, 9.7 \%$, and $17.2 \%$, respectively. These lesions were frequently located at pyriform sinus or in the posterior wall of the pharynx (70\%, 37/53 lesions). Most of the lesions were detected at an early stage, though 4 lesions were associated with lymph node metastasis when their primary sites were detected ( 1 postcricoid area, 2 posterior wall of hypopharynx, and 1 lateral wall of oropharynx).

Conclusions: Patients with esophageal SCC should undergo careful inspection of the pyriform sinus and posterior wall of the pharynx for detection of H\&N SCCs. Methods to open the hypopharyngeal space, such as the Valsalva maneuver, should be included in the surveillance program.

mary H\&N cancers was provided. However, detailed analyses of second primary H\&N cancers may help to elucidate the characteristics of cancers caused by the field cancerization effect, and thus help to establish effective surveillance methods for patients with esophageal cancer. In this study, we therefore investigated the incidence, primary sites, and stages of second primary H\&N SCCs detected in patients with esophageal SCC.

\section{Patients and methods}

$\nabla$

\section{Patients}

A total of 818 patients underwent endoscopic resection for superficial esophageal cancer at Osaka Medical Center for Cancer and Cardiovascular Diseases between January 2006 and December 2013. We prospectively collected baseline clinical data for these patients, including treatment date, age, sex, past medical history, and lesion characteristics, such as location, size, histology, and depth of invasion. From this database, we identified 439 patients who met the following inclusion criteria: 
(1) histologically proven esophageal SCC; (2) mucosal or submucosal esophageal SCC; (3) no prior radiotherapy, chemotherapy, or surgery for esophageal SCC; (4) no prior diagnosis or treatment of H\&N SCC; (5) no history of other incurable cancer before endoscopic resection; and (6) followed up with upper gastrointestinal endoscopy in our hospital for more than 6 months (○ Fig. 1)

In this study, multiple H\&N cancers detected after endoscopic resection were classified as metachronous. The incidences and characteristics of metachronous H\&N cancers were obtained from electronic medical records. All the lesions were histologically confirmed as SCC according to World Health Organization criteria [12]. The lesions were also diagnosed as cancerous based on obvious cytological abnormalities of the squamous epithelium, even when the abnormalities were confined to the lower half of the squamous epithelium [13]. This study was approved by the ethics committee at Osaka Medical Center for Cancer and Cardiovascular Diseases.

\section{Follow up}

Patients were followed up at our hospital or by the referring physicians after treatment. In this study, we only analyzed second primary H\&N cancers in patients who were followed up for more than 6 months in our hospital, to ensure the quality of endoscopic examination.

Surveillance of H\&N cancers was conducted by upper gastrointestinal endoscopy and pharyngolaryngoscopy. Upper gastrointestinal endoscopy was generally conducted by gastroenterologists at 2 months after endoscopic resection, and annually thereafter. Most endoscopic examinations were carried out using a magnifying endoscope with narrow-band imaging (NBI) (GIFQ240Z or GIF-H260Z; Olympus Optical Co Ltd, Tokyo, Japan) fitted with a soft black hood attachment (MB-162 for GIF-Q240Z, or MB-46 for GIF-H260Z; Olympus) on its top. Pharyngolaryngoscopy by otorhinolaryngologist was conducted annually or as dictated by symptoms such as hoarseness or discomfort in swallowing. During endoscopic examination, we observed the oropharynx, hypopharynx, and oral cavity. If a well-demarcated brownish area and microvascular irregularities were observed with $\mathrm{NBI}$, the lesion was diagnosed as endoscopically suspected superficial SCC [14], and biopsy samples were taken.

\section{General treatment principles of H\&N cancer}

All detected H\&N cancers were treated at our hospital based on a strategy determined by otorhinolaryngologists and gastroenterologists. Lesions that fulfilled the following criteria were treated by minimally invasive treatment, such as transoral surgical mucosectomy or endoscopic resection: (1) cancers limited to the epithelium or invading into the surface part of the subepithelial layer; (2) cancers with no spread into the pharyngeal space bilaterally; and (3) no lymph node or distant metastasis visible by computed tomography. Because lesions in the oral cavity and superior wall of the oropharynx can be accessed easily by a surgical device, transoral surgical mucosectomy was carried out by otorhinolaryngologists. However, if a transoral direct approach was difficult, endoscopic resection was performed by gastrointestinal endoscopists. If the detected lesion did not meet the indication criteria for minimally invasive treatment as mentioned above, radical surgery, radiotherapy, or chemoradiotherapy was considered.

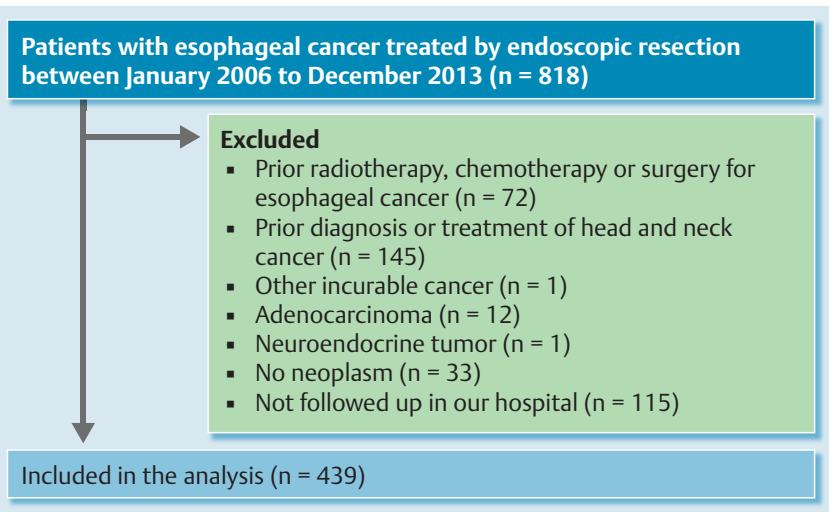

Fig. 1 Flowchart of patients.

\section{Statistical analysis}

The cumulative incidence rates of metachronous H\&N cancers were plotted using the Kaplan-Meier method. The observation period was measured from the date of endoscopic resection to the date of detection of metachronous multiple cancers, or the latest endoscopic examination in patients in whom multiple cancers were not found. Categorical variables were compared using Yates $\mathrm{X}^{2}$ tests. For all analyses, a two-sided $P$ value $<0.05$ was considered statistically significant. Statistical analyses were performed using JMP version 10.0 (SAS Institute, Cary, NC, USA).

\section{Results}

\section{$\nabla$}

The characteristics of the 439 patients and the esophageal lesions are listed in 0 Table1. For patients with synchronous multiple esophageal SCCs, data for the deepest or largest lesion are shown. The patients included 370 men and 69 women, with a median age of 68 years (range $41-83$ ). Eighteen patients had submucosal cancer with lymphovascular involvement, 42 had submucosal cancer without lymphovascular involvement, and 23 had mucosal cancer with lymphovascular involvement. Of these 83 patients, additional treatment was performed in 71 patients $(63$ chemoradiotherapy, 7 surgery, and 1 chemotherapy) to reduce the risk of lymph node metastasis. The remaining 12 patients did not receive the additional treatment because of patient refusal in 11 cases and liver cirrhosis in 1 case.

Among all the patients, 98\% (428/439) received follow-up examinations at 1 year, $79 \%(346 / 439)$ at 2 years, $60 \%(264 / 439)$ at 3 years, $44 \%(191 / 439)$ at 4 years, and $28 \%(123 / 439)$ at 5 years. A total of 53 metachronous $\mathrm{H} \& \mathrm{~N}$ cancers developed in 40 patients after median follow-up of 46 months (range 9-109), with cumulative incidence rates of metachronous $\mathrm{H} \& \mathrm{~N}$ cancers at 3,5 , and 7 years of $5.3 \%, 9.7 \%$, and $17.2 \%$, respectively ( $\bullet$ Fig. 2 ). The characteristics of the metachronous H\&N cancers are listed in $\bullet$ Table 2. Thirty-five lesions were located in the hypopharynx, 9 in the oropharynx, 4 in the oral cavity, and 5 in the larynx. Four lesions were detected by otorhinolaryngologists ( 1 lateral wall of oropharynx, 2 oral floor, and 1 glottis), and all of the remaining lesions were detected by gastroenterologists. Among all the subsites in the H\&N region, $70 \%$ of lesions were located at the pyriform sinus $(47 \%, 25 / 53)$ or posterior wall of the pharynx $(23 \%$, $12 / 53)$. The median size of the detected lesions was $17 \mathrm{~mm}$ (range $2-45 \mathrm{~mm}$ ). Minimally invasive treatment such as endoscopic resection or transoral surgical mucosectomy was conducted for 39 of the 53 lesions (74\%). O Table 3 shows the $\mathrm{T}$ and $\mathrm{N}$ 
Table 1 Characteristics of patients and lesions.

\begin{tabular}{|c|c|}
\hline Gender, male/female, $\mathrm{n}$ & $370 / 69$ \\
\hline Age, median (range), y & $68(41-83)$ \\
\hline \multicolumn{2}{|l|}{ Lesion location, $\mathrm{n}$} \\
\hline Cervical esophagus & 51 \\
\hline Upper thoracic esophagus & 115 \\
\hline Middle thoracic esophagus & 197 \\
\hline Lower thoracic esophagus & 76 \\
\hline Lesion size, median (range), mm & $12(2-80)$ \\
\hline \multicolumn{2}{|l|}{ Tumor depth, $\mathrm{n}$} \\
\hline EP & 118 \\
\hline LPM & 171 \\
\hline MM & 90 \\
\hline SM & 60 \\
\hline \multicolumn{2}{|l|}{ Lymphovascular involvement, $\mathrm{n}$} \\
\hline Positive & 41 \\
\hline Negative & 398 \\
\hline
\end{tabular}

EP, epithelium; LPM, lamina propria; MM, muscularis mucosa; SM, submucosa.

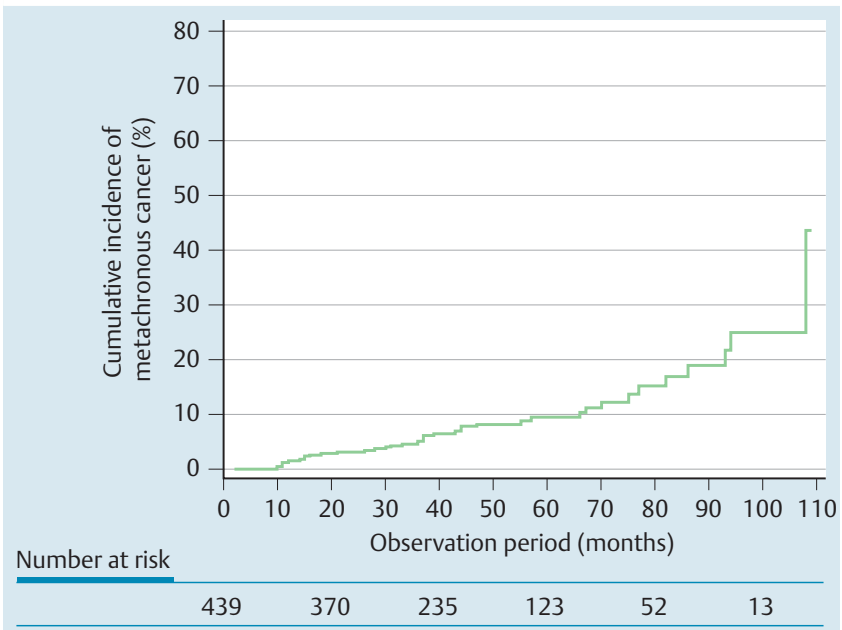

Fig. 2 Cumulative incidence of metachronous head and neck cancers after endoscopic resection of esophageal squamous cell carcinoma.

stages of the detected cancers (no distant metastasis was detected in any of the lesions). Most of the lesions (70\%, 37/53) were classified as Tis/T1N0, although 4 lesions had lymph node metastasis when their primary sites were detected (1 postcricoid area, 2 posterior wall of hypopharynx, and 1 lateral wall of orophar$\mathrm{ynx})$. Three of 5 protruded lesions and 1 of 46 flat lesions had lymph node metastasis. There was a significant association $(P=$ 0.0002 ) between endoscopic appearance (protruded or flat) and $\mathrm{N}$ stage (N0 or $\mathrm{N} 1-2$ ).

\section{Discussion}

$\nabla$

In this study, we demonstrated a cumulative 5-year incidence rate of $9.7 \%$ for metachronous H\&N SCC in patients with esophageal SCC. The pyriform sinus and posterior wall of the pharynx were the most common sites for the development of metachronous SCCs. Most of the lesions were detected at an early stage and were completely removed by minimally invasive treatment, such as transoral surgical mucosectomy or endoscopic resection. However, some lesions in the postcricoid area and posterior wall of the hypopharynx were initially diagnosed at an advanced stage.
Table 2 Characteristics of metachronous head and neck cancers.

\begin{tabular}{|c|c|}
\hline Patients, n (\%) & $40(9.1)$ \\
\hline Lesions, $\mathrm{n}$ & 53 \\
\hline \multicolumn{2}{|l|}{ Lesion location, $\mathrm{n}$} \\
\hline \multicolumn{2}{|l|}{ Hypopharynx } \\
\hline Left pyriform sinus & 10 \\
\hline Right pyriform sinus & 15 \\
\hline Postcricoid area & 3 \\
\hline Posterior wall & 7 \\
\hline \multicolumn{2}{|l|}{ Oropharynx } \\
\hline Superior wall & 3 \\
\hline Posterior wall & 5 \\
\hline Lateral wall & 1 \\
\hline \multicolumn{2}{|l|}{ Oral cavity } \\
\hline Oral floor & 2 \\
\hline Buccal mucosa & 2 \\
\hline \multicolumn{2}{|l|}{ Larynx } \\
\hline Supraglottis & 4 \\
\hline Glottis & 1 \\
\hline Lesion size, median (range), mm & $17(2-45)$ \\
\hline \multicolumn{2}{|l|}{ Macroscopic appearance, $\mathrm{n}^{1}$} \\
\hline Flat & 46 \\
\hline Protruded & 5 \\
\hline \multicolumn{2}{|l|}{ Treatment, $\mathrm{n}$} \\
\hline Endoscopic resection & 31 \\
\hline Transoral surgical mucosectomy & 8 \\
\hline Radical surgery & 3 \\
\hline Radiotherapy & 4 \\
\hline Chemoradiotherapy & 3 \\
\hline Endoscopic resection \& radiotherapy & 1 \\
\hline Surgery \& radiotherapy & 1 \\
\hline Surgery \& chemoradiotherapy & 1 \\
\hline Observation ${ }^{2}$ & 1 \\
\hline
\end{tabular}

${ }^{1}$ Macroscopic types of 2 lesions were not recorded

${ }^{2}$ Lesions were not recorded ${ }^{2}$ because of other incurable cancer

Table 3 T and $\mathrm{N}$ stages of metachronous head and neck cancers.

\begin{tabular}{|lllll|}
\hline \multicolumn{1}{|l}{ TN stage, $\mathbf{n}$} & & \\
\hline Lesion location & Tis/T1N0 & T2N0 & T3NO & TanyN1-2 \\
\hline Hypopharynx & & & & \\
\hline Pyriform sinus & 17 & 7 & 1 & 0 \\
\hline Postcricoid area & 2 & 0 & 0 & 1 \\
\hline Posterior wall & 5 & 0 & 0 & 2 \\
\hline Oropharynx & & & & \\
\hline Superior wall & 2 & 1 & 0 & 0 \\
\hline Posterior wall & 4 & 1 & 0 & 0 \\
\hline Lateral wall & 0 & 0 & 0 & 1 \\
\hline Oral cavity & 2 & 2 & 0 & 0 \\
\hline Larynx & 5 & 0 & 0 & 0 \\
\hline
\end{tabular}

In Japan, the age-specific annual incidence rate for oral and pharyngeal cancer in 65- to 69-year-olds was 30.6 per 100,000 in 2008 [15]. Compared with the general population, our study demonstrated that patients with esophageal cancer were at extremely high risk of developing $\mathrm{H} \& \mathrm{~N}$ cancers, with an incidence of approximately 2000 per 100,000 patients. We also previously reported that metachronous esophageal cancer frequently developed in patients who had undergone endoscopic resection for esophageal cancer (cumulative incidence rate at 5 years of 20.6\%) [16]. These studies demonstrate that the entire epithelial surface of the upper digestive tract is at increased risk of cancer in 


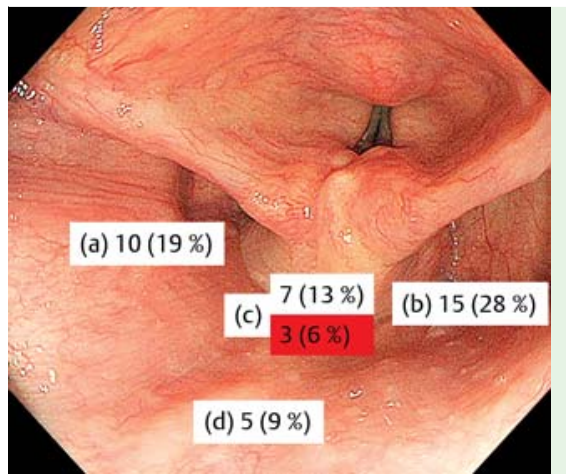

Fig. 3 Number (prevalence [\%]) of cancers in each subsite. a left pyriform sinus. $\mathbf{b}$ right pyriform sinus. c postcricoid area and posterior wall of the hypopharynx. $\mathbf{d}$ posterior wall of the oropharynx. Number (prevalence [\%]) with a red background indicates cancers with lymph node metastasis.

patients with esophageal cancer, thus supporting the field cancerization theory.

According to the H\&N cancer registry in Japan, a total of 3899 H\&N cancers were newly diagnosed in 2012,840 of which (22\%) were classified as Tis/T1N0, and 249 (6.4\%) of which were treated by minimally invasive methods, such as transoral surgical mucosectomy or endoscopic resection [17]. In this study using periodic endoscopic surveillance with NBI, 70\% (37/53) of lesions were detected as Tis/T1N0 cancers, and 39 of the 53 lesions (74\%) were treated by minimally invasive procedures. These results indicate that periodic endoscopic surveillance facilitates the early detection of H\&N cancers, and suggest that NBI may also be relevant [18].

Effective surveillance requires an understanding of the detailed features, such as the annual incidence and common sites of H\&N cancers. Katada et al. reported the prevalence of superficial H\&N cancers in patients with esophageal cancer, but not their annual incidence [10]. Hori et al. investigated the annual incidence of H\&N cancers, but the number of lesions was relatively small and their detailed locations were not reported [9]. The H\&N cancer registry in Japan shows the detailed locations of the cancers, but most cancers included in the registry are advanced cases [17]. The current study is thus the largest to demonstrate the incidence of superficial H\&N cancers in patients with esophageal cancer, with detailed information on the locations and stages of the lesions. Although this study was conducted in patients with esophageal cancer, the findings regarding cancer location might be applied to cancers arising based on the field cancerization theory.

In this study, H\&N cancers were frequently located at the pyriform sinus or posterior wall of the pharynx (70\%, 37/53 lesions). Because the structure of the H\&N region is complex and it is difficult to view the region thoroughly, information on the most common sites of $\mathrm{H} \& \mathrm{~N}$ cancers may help effective surveillance. Although we detected most H\&N lesions at an early phase, some lesions in the postcricoid area and posterior wall of the hypopharynx already had lymph node metastasis when their primary sites were discovered. These anatomically adjacent sites in the hypopharynx constitute a closed space, which disturbs us to notice subtile changes in the muscosa during endoscopic observation ( $\bullet$ Fig.3). Methods to open this closed space, such as the Valsalva maneuver, would facilitate detection of these lesions, and such methods should thus be included as a part of the routine surveillance program.

Our study was limited by its retrospective, single-center design, and by the large number of patients (115 patients) who were lost to follow up. However, conducting a multicenter study would be difficult given that there is currently no established surveil- lance program for $\mathrm{H} \& \mathrm{~N}$ cancer. Furthermore, limiting the patients who were followed up at our hospital allowed us to maintain similar surveillance intervals and similar qualities of endoscopic examinations for H\&N cancer. Despite the limitations, the results of this study will thus contribute to the development of future surveillance programs.

In conclusion, the 5-year incidence of metachronous H\&N SCC in patients with esophageal SCC was $9.7 \%$, with the pyriform sinus and posterior wall of the pharynx being the most common sites. A standardized surveillance program for H\&N SCC should be developed for patients with esophageal SCC.

\section{Competing interests: None}

\section{References}

1 Matsubara T, Yamada K, Nakagawa A. Risk of second primary malignancy after esophagectomy for squamous cell carcinoma of the thoracic esophagus. J Clin Oncol 2003; 21: 4336-4341

2 Ishihara $\mathrm{R}$, Tanaka $\mathrm{H}$, Iishi $\mathrm{H}$ et al. Long-term outcome of esophageal mucosal squamous cell carcinoma without lymphovascular involvement after endoscopic resection. Cancer 2008; 112: 2166-2172

3 Slaughter DP, Southwick HW, Smejkal W. Field cancerization in oral stratified squamous epithelium: clinical implication of multicentric origin. Cancer 1953; 6: 963-968

4 Akira Y, Watanabe H, Fukuda $\mathrm{H}$ et al. Multiple cancers associated with esophageal and oropharyngolaryngeal squamous cell carcinoma and the aldehyde dehydrogenase-2 genotype in male Japanese drinkers. Cancer Epidemiol Biomarkers Prev 2002; 11: 895-900

5 Watanabe A, Hsokawa M, Taniguchi $M$ et al. Imapact of endoscopic screening on early detection of hypopharyngeal cancer. Head Neck 2006; 28: $350-354$

6 Muto M, Satake H, Yano T et al. Long-term outcome of transoral organpreserving pharyngeal endoscopic resection for superficial pharyngeal cancer. Gastrointest Endosc 2011; 74: 477-484

7 Satake H, Yano T, Muto M et al. Clinical outcome after endoscopic resection for superficial pharyngeal squamous cell carcinoma invading the subepithelial layer. Endoscopy 2015; 47: 11 -18

8 Hanaoka $N$, Ishihara $R$, Takeuchi $Y$ et al. Endoscopic submucosal dissection as minimally invasive treatment for superficial pharyngeal cancer: phase II study (with video). Gastrointest Endosc (Epub 2015 Jul 30)

9 Hori K, Okada H, Kawahara Y et al. Lugol-voiding lesions are an important risk factor for a second primary squamous cell carcinoma in patients with esophageal cancer or head and neck cancer. Am J Gastroenterol 2011; 106: 858-866

10 Katada C, Tanabe S, Koizumi $W$ et al. Narrow band imaging for detecting superficial squamous cell carcinoma of the head and neck in patients with esophageal cell carcinoma. Endoscopy 2010; 42: 185-190

11 Shimizu Y, Tsukagoshi H, Fujita $M$ et al. Head and neck cancer arising after endoscopic mucosal resection for squamous cell carcinoma of the esophagus. Endoscopy 2003; 35: $322-326$

12 Barnes D, Eveson JW, Reichart P et al. World Health Organization Classification of Tumors. Pathology and genetics. Head and neck tumors. Lyon: IARC Press; 2005

13 Japan Esophageal Society. Japanese Classification of Esophageal Cancer, tenth edition: parts II and III. Esophagus 2009; 6: 71-94

14 Muto M, Nakane M, Katada C et al. Squamous cell carcinoma in situ at oropharyngeal and hypopharyngeal mucosal sites. Cancer 2004; 101 : $1375-1381$

15 Matsuda A, Matsuda T, Shibata A et al. Cancer incidence rates in Japan in 2008: A study of 25 population-based cancer registries for the monitoring of cancer incidence in Japan (MCIJ) project. Jpn J Clin Oncol 2014; 44: 388-396

16 Yamashina T, Ishihara R, Nagai $K$ et al. Long-term outcome and metastatic risk after endoscopic resection of superficial esophageal squamous cell carcinoma. Am J Gastroenterol 2013; 108: 544-551

17 Japan Society for Head and Neck Cancer. Report of head and neck cancer registry of Japan, clinical statistics of registered patients, 2012.Jpn J Head Neck Cancer 2014; 40: 1-14

18 Muto M, Minashi K, Yano T et al. Early detection of superficial squamous cell carcinoma in the head and neck region and esophagus by narrow band imaging: a multicenter randomized controlled trial. J Clin Oncol 2010; 28: 1566-1572 